\title{
Topological systems as a framework for institutions
}

\author{
Jeffrey T. Denniston ${ }^{\mathrm{a}}$, Austin Melton ${ }^{\mathrm{b}}$, Stephen E. Rodabaugh ${ }^{\mathrm{c}}$, Sergey A. Solovyov ${ }^{\text {坟 }}$ \\ ${ }^{a}$ Department of Mathematical Sciences, Kent State University \\ Kent, Ohio, USA 44242 \\ ${ }^{b}$ Departments of Computer Science and Mathematical Sciences, Kent State University \\ Kent, Ohio, USA 44242 \\ ${ }^{c}$ College of Science, Technology, Engineering, Mathematics (STEM), Youngstown State University \\ Youngstown, Ohio, USA 44555-3347 \\ ${ }^{d}$ Institute of Mathematics, Faculty of Mechanical Engineering, Brno University of Technology, Technicka 2896/2, 616 69 \\ Brno, Czech Republic
}

\begin{abstract}
Recently, J. T. Denniston, A. Melton, and S. E. Rodabaugh introduced a lattice-valued analogue of the concept of institution of J. A. Goguen and R. M. Burstall, comparing it, moreover, with the (lattice-valued version of the) notion of topological system of S. Vickers. In this paper, we show that a suitable generalization of topological systems provides a convenient framework for doing certain kinds of (lattice-valued) institutions.
\end{abstract}

Keywords: adjoint situation, affine theory, comma category, elementary institution, localification and spatialization procedure, topological institution, topological space, topological system, variety of algebras 2010 MSC: 18A25, 18B15, 18B30, 18B99, 18C10

\section{Introduction}

There exists a convenient approach to logical systems in computer science, which is based in the concept of institution of J. A. Goguen and R. M. Burstall [24]. An institution comprises a category of (abstract) signatures, where every signature has its associated sentences, models, and a relationship of satisfaction; this relationship is invariant (in a certain sense) under change of signature. The slogan, therefore, is "truth is invariant under change of notation". Examples of institutions include unsorted universal algebra, manysorted algebra, order-sorted algebra, several variants of first-order logic, partial algebra (see, e.g., 23]). More examples can be found in [13, Subsection 3.2]. A number of authors (including the initiators themselves) have proposed generalizations of institutions in various forms as well as advanced their theories 25, 26, 34, 35, 40]. Moreover, some authors used a purely category-theoretic approach to institutions (see, e.g., 12]).

There exists the concept of topological system of S. Vickers [49], which is based in the ideas of geometric logic [50] and intended to provide a common setting for both topological spaces (point-set topology) and their underlying algebraic structures - locales (point-free topology). In particular, S. Vickers presented system spatialization and localification procedures, which created ways to move back and forth between the categories of topological spaces (resp., locales) and topological systems. Recently, the latter concept has gained in interest in connection with lattice-valued topology. In particular, 9, 10] introduced and studied the notion of lattice-valued topological system; 27] discovered a convenient relationship between crisp and lattice-valued topology, based in topological systems; and [43, 47] studied a lattice-valued analogue of the above-mentioned system spatialization procedure.

\footnotetext{
The author gratefully acknowledges the support of Grant Agency of Czech Republic (GAČR) and Austrian Science Fund (FWF) within bilateral project No. I 1923-N25 "New Perspectives on Residuated Posets".

Email addresses: jdennist@kent.edu (Jeffrey T. Denniston), amelton@kent.edu (Austin Melton), serodabaugh@ysu.edu (Stephen E. Rodabaugh), solovjovs@fme.vutbr.cz (Sergey A. Solovyov ${ }^{\text {th }}$ )
} 
In an attempt to find possible relationships between institutions and topological systems, at the 35th Linz Seminar on Fuzzy Set Theory, J. T. Denniston, A. Melton, and S. E. Rodabaugh [11] presented a latticevalued analogue of institutions, and showed that (lattice-valued) topological systems provide a particular instance of the latter. Moreover, 42] introduced (crisp) topological institutions, based in topological systems, the slogan being that "the central concept is the theory, not the formula". To continue this line of study, several authors considered some other institutional modifications (e.g., probability institutions, quantum institutions, etc.) [5, 8], motivated by the ideas of quantum logic (in connection with quantum physics).

We notice that some researchers prefer the reverse of the above slogan. While the theory plays an important role in building logic, the terms should be constructed first (to allow variable substitution), and after that sentences should be constructed on terms to get a sentence functor. The construction of terms through the term monad plays a key role in allowing variable substitution and variable assignment. Having only abstract categories for signatures hides the use of variables and the difference between terms and sentences. Term and sentence construction are two separate processes, which should be revealed together with the process of variable assignment. The readers with the same point of view could look into 18, 19, 20, 21, 22] for a particular fuzzy approach to terms and their respective monad. We notice, however, that although the main point of institution theory is exactly to liberate the logic study from explicit variables and substitutions, when needed institution theory has its well established approach to these 13].

The main purpose of this paper is to show that a suitably generalized concept of topological system makes a setting for a particular type of (lattice-valued) institutions, namely, elementary institutions [41, 42]. In so doing, we aim at providing a convenient framework for building up the theory of lattice-valued institutions. More precisely, there already exists a well-developed theory of lattice-valued (also many-valued or fuzzy) logic, which has been given a coherent statement by P. Hájek in [28], and which by now has much diversified w.r.t. the algebraic structures (which often constitute a variety) over which the respective fuzzification is done. The concept of institution, however, being a significant part of the crisp logical developments (see, e.g., [13]), has been fuzzified just recently in [11]. With this article, we are going to extend further this fuzzification in a way, which could encompass various lattice-valued frameworks. We achieve this goal with a modification of the affine context of Y. Diers [14, 15, 16], which is based in an arbitrary variety of algebras, thereby providing a unifying setting for many possible fuzzifications of institutions (which are to come), each of them based in the favourite variety of it's authors (e.g., a variety of residuated lattices). The main advantage of such a unifying setting is the fact that every statement, which is proved in the affine framework (namely, for all varieties) will be valid for each particular fuzzification (namely, for each particular variety).

\section{Affine systems and their related tools}

This section reviews the notions of affine systems and spaces, as well as their related spatialization and localification procedures (for details, see [46, 47, 48]). Since the localification procedure has not yet appeared in the literature in full detail, we provide a more thorough description. We conclude this section with the approach to topological systems, which is motivated by algebraic theories of F. W. Lawvere [33].

A particular remark is helpful w.r.t. the system terminology of this paper. Following the notion of lattice-valued topological system of [9, 10], in [4] is provided a more general concept under the name of variety-based topological system, which eventually gave rise to the notion and theory of categoricallyalgebraic topology 44|. It was subsequently discovered that the latter concept had already been introduced by Y. Diers [14, 15, 16] under the name of affine (or algebraic) set, but in a quite different context with no lattice-valued motivation or system notion. As a consequence our generalized topological spaces (resp., systems) are renamed affine spaces (resp., systems).

\subsection{Algebraic preliminaries}

In this subsection, we recall the algebraic notions which will be used throughout the paper.

Definition 1. Let $\Omega=\left(n_{\lambda}\right)_{\lambda \in \Lambda}$ be a family of cardinal numbers, which is indexed by a (possibly proper or empty) class $\Lambda$. An $\Omega$-algebra is a pair $\left(A,\left(\omega_{\lambda}^{A}\right)_{\lambda \in \Lambda}\right)$, which comprises a set $A$ and a family of maps 
$A^{n_{\lambda}} \stackrel{\omega_{\lambda}^{A}}{\longrightarrow} A\left(n_{\lambda}\right.$-ary primitive operations on $\left.A\right)$. An $\Omega$-homomorphism $\left(A,\left(\omega_{\lambda}^{A}\right)_{\lambda \in \Lambda}\right) \stackrel{\varphi}{\rightarrow}\left(B,\left(\omega_{\lambda}^{B}\right)_{\lambda \in \Lambda}\right)$ is a map $A \stackrel{\varphi}{\longrightarrow} B$, which makes the diagram

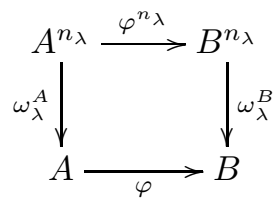

commute for every $\lambda \in \Lambda$. $\mathbf{A l g}(\Omega)$ is the construct of $\Omega$-algebras and $\Omega$-homomorphisms.

Definition 2. Let $\mathcal{M}$ (resp., $\mathcal{E}$ ) be the class of $\Omega$-homomorphisms with injective (resp., surjective) underlying maps. A variety of $\Omega$-algebras is a full subcategory of $\mathbf{A} \lg (\Omega)$, which is closed under the formation of products, $\mathcal{M}$-subobjects (subalgebras), and $\mathcal{E}$-quotients (homomorphic images). The objects (resp., morphisms) of a variety are called algebras (resp., homomorphisms).

In the following, we provide some examples of varieties, which are relevant to this paper.

\section{Example 3.}

(1) $\operatorname{CSLat}(\bigvee)$ is the variety of $\bigvee$-semilattices, i.e., partially ordered sets, which have arbitrary joins, and $\operatorname{CSLat}(\bigwedge)$ is the variety of $\bigwedge$-semilattices, i.e., partially ordered sets, which have arbitrary meets. $\operatorname{CSLat}(\bigvee)(\operatorname{resp} . \operatorname{CSLat}(\bigwedge))$ is precisely the category $\mathbf{A l g}(\bigvee)(\operatorname{resp} . \mathbf{A l g}(\bigwedge))$

(2) Frm is the variety of frames, i.e., $\bigvee$-semilattices $A$, with singled out finite meets, and which additionally satisfy the distributivity condition $a \wedge(\bigvee S)=\bigvee_{s \in S}(a \wedge s)$ for every $a \in A$ and every $S \subseteq A[32$. Frm is a full subcategory of $\mathbf{A l g}(\bigvee, \wedge)$.

(3) CBAlg is the variety of complete Boolean algebras, i.e., complete lattices $A$ such that $a \wedge(b \vee c)=$ $(a \wedge b) \vee(a \wedge c)$ for every $a, b, c \in A$, equipped with a unary operation $A \stackrel{(-)^{*}}{\longrightarrow} A$ such that $a \vee a^{*}=\top_{A}$ and $a \wedge a^{*}=\perp_{A}$ for every $a \in A$, where $\top_{A}$ (resp., $\perp_{A}$ ) is the largest (resp., smallest) element of $A$. CBAlg is a full subcategory of $\operatorname{Alg}\left(\bigvee, \bigwedge,{ }^{*}\right)$.

(4) CSL is the variety of closure semilattices, i.e., ^-semilattices, with the singled out bottom element. CSL is a full subcategory of $\operatorname{Alg}(\bigwedge, \perp)$.

\subsection{Affine spaces}

In this subsection, we provide an extension of the notion of affine set of Y. Diers [14, 15, 16].

Definition 4. Given a functor $\mathbf{X} \stackrel{T}{\rightarrow} \mathbf{B}^{o p}$, where $\mathbf{B}$ is a variety of algebras, $\mathbf{A f} \mathbf{S p c}(T)$ is the concrete category over $\mathbf{X}$, whose objects (T-affine spaces or $T$-spaces) are pairs $(X, \tau)$, where $X$ is an $\mathbf{X}$-object and $\tau$ is a B-subalgebra of $T X$; and whose morphisms (T-affine morphisms or $T$-morphisms) $\left(X_{1}, \tau_{1}\right) \stackrel{f}{\rightarrow}\left(X_{2}, \tau_{2}\right)$ are $\mathbf{X}$-morphisms $X_{1} \stackrel{f}{\rightarrow} X_{2}$ with the property that $(T f)^{o p}(\alpha) \in \tau_{1}$ for every $\alpha \in \tau_{2}$.

The following easy result will give rise to our main examples of $T$-spaces and $T$-morphisms.

Proposition 5. Given a variety $\mathbf{B}$, every subcategory $\mathbf{S}$ of $\mathbf{B}^{o p}$ induces a functor Set $\times \mathbf{S} \stackrel{\mathcal{P}_{\mathbf{S}}}{\longrightarrow} \mathbf{B}^{o p}$, $\mathcal{P}_{\mathbf{S}}\left(\left(X_{1}, B_{1}\right) \stackrel{(f, \varphi)}{\longrightarrow}\left(X_{2}, B_{2}\right)\right)=B_{1}^{X_{1}} \stackrel{\mathcal{P}_{\mathbf{S}}(f, \varphi)}{\longrightarrow} B_{2}^{X_{2}}$, where $\left(\mathcal{P}_{\mathbf{S}}(f, \varphi)\right)^{o p}(\alpha)=\varphi^{o p} \circ \alpha \circ f$.

The case $\mathbf{S}=\left\{B \stackrel{1_{B}}{\longrightarrow} B\right\}$ provides a functor Set $\stackrel{\mathcal{P}_{B}}{\longrightarrow} \mathbf{B}^{o p}, \mathcal{P}_{B}\left(X_{1} \stackrel{f}{\rightarrow} X_{2}\right)=B^{X_{1}} \stackrel{\mathcal{P}_{B} f}{\longrightarrow} B^{X_{2}}$, where $\left(\mathcal{P}_{B} f\right)^{o p}(\alpha)=\alpha \circ f$. In particular, if $\mathbf{B}=\mathbf{C B A l g}$, and $\mathbf{S}=\left\{2 \stackrel{1_{2}}{\longrightarrow} 2\right\}$, then one obtains the well-known contravariant powerset functor Set $\stackrel{\mathcal{P}}{\rightarrow} \mathbf{C B A l g}^{o p}$, which is given on a map $X_{1} \stackrel{f}{\rightarrow} X_{2}$ by $\mathcal{P} X_{2} \stackrel{(\mathcal{P} f)^{o p}}{\rightarrow} \mathcal{P} X_{1}$ with $(\mathcal{P} f)^{o p}(S)=\left\{x \in X_{1} \mid f(x) \in S\right\}$.

The following examples of the categories of the form $\operatorname{Af} \mathbf{S p c}(T)$ will be relevant to this paper. 


\section{Example 6.}

(1) If $\mathbf{B}=\mathbf{F r m}$, then $\mathbf{A f} \operatorname{Spc}\left(\mathcal{P}_{2}\right)$ is the category Top of topological spaces.

(2) If $\mathbf{B}=\mathbf{C S L}$, then $\mathbf{A f} \mathbf{S p c}\left(\mathcal{P}_{2}\right)$ is the category Cls of closure spaces 4$]$.

(3) $\operatorname{Af} \operatorname{Spc}\left(\mathcal{P}_{B}\right)$ is the category $\operatorname{AfSet}(B)$ of affine sets of Y. Diers.

(4) If $\mathbf{B}=\mathbf{F r m}$, then $\mathbf{A f} \operatorname{Spc}\left(\mathcal{P}_{\mathbf{S}}\right)$ is the category $\mathbf{S}$-Top of variable-basis lattice-valued topological spaces of S. E. Rodabaugh [38].

Given a map $X \stackrel{f}{\rightarrow} Y$ and subsets $R \subseteq X, S \subseteq Y, f^{\rightarrow}(R):=\{f(r) \mid r \in R\}$ and $f^{\leftarrow}(S):=\{x \mid f(x) \in$ $S\}$ [39]; this notation will be employed through the rest of the paper.

All of the categories of the form $\operatorname{AfSpc}(T)$ share the following convenient property.

Theorem 7. Given a functor $\mathbf{X} \stackrel{T}{\rightarrow} \mathbf{B}^{o p}$, the concrete category $(\mathbf{A f} \mathbf{S p c}(T),|-|)$ is topological (over $\left.\mathbf{X}\right)$.

Proof. Given a $|-|$-structured source $\mathcal{S}=\left(X \stackrel{f_{i}}{\longrightarrow}\left|\left(X_{i}, \tau_{i}\right)\right|\right)_{i \in I}$, its lift on $X$ w.r.t. $\mathcal{S}$ is given by the subalgebra of $T X$, which is generated by the union $\bigcup_{i \in I}\left(\left(T f_{i}\right)^{o p}\right) \rightarrow\left(\tau_{i}\right)$; it is left to the reader to show that this lift is the unique initial lift. Alternatively, given a $|-|$-structured sink $\mathcal{S}=\left(\left|\left(X_{i}, \tau_{i}\right)\right| \stackrel{f_{i}}{\longrightarrow} X\right)_{i \in I}$, its lift on $X$ w.r.t. $\mathcal{S}$ is the intersection $\bigcap_{i \in I}\left(\left(T f_{i}\right)^{o p}\right)^{\leftarrow}\left(\tau_{i}\right)$; it is left to the reader to show that this lift is the unique final lift.

As a consequence, one obtains the well-known result that all the categories of Example 6 are topological.

\subsection{Affine systems}

Following the ideas of [47, 48], this subsection introduces the concept of affine system as an analogue of topological systems of S. Vickers [49].

Definition 8. Given a functor $\mathbf{X} \stackrel{T}{\rightarrow} \mathbf{B}^{o p}, \mathbf{A f} \mathbf{S y s}(T)$ is the comma category $\left(T \downarrow 1_{\mathbf{B}^{o p}}\right)$, which is concrete over the product category $\mathbf{X} \times \mathbf{B}^{o p}$, whose objects (T-affine systems or $T$-systems) are triples $(X, \kappa, B)$, which are made with $\mathbf{B}^{o p}$-morphisms $T X \stackrel{\kappa}{\rightarrow} B$; and whose morphisms (T-affine morphisms or $T$-morphisms) $\left(X_{1}, \kappa_{1}, B_{1}\right) \stackrel{(f, \varphi)}{\longrightarrow}\left(X_{2}, \kappa_{2}, B_{2}\right)$ are $\mathbf{X} \times \mathbf{B}^{o p}$-morphisms $\left(X_{1}, B_{1}\right) \stackrel{(f, \varphi)}{\longrightarrow}\left(X_{2}, B_{2}\right)$, which make the diagram

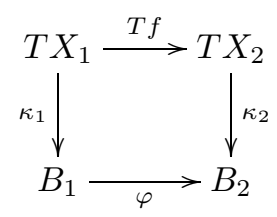

commute.

\section{Example 9.}

(1) If $\mathbf{B}=\mathbf{F r m}$, then $\operatorname{Af} \operatorname{Sys}\left(\mathcal{P}_{2}\right)$ is the category TopSys of topological systems of S. Vickers.

(2) If $\mathbf{B}=\mathbf{S e t}$, then $\mathbf{A f} \operatorname{Sys}\left(\mathcal{P}_{B}\right)$ is the category $\mathbf{C h u} \mathbf{u}_{B}$ of Chu spaces over a set $B$ of P.-H. Chu [7].

(3) If $\mathbf{B}=\mathbf{F r m}$, then $\mathbf{A f} \operatorname{Sys}\left(\mathcal{P}_{\mathbf{S}}\right)$ is the category $\mathbf{S}$-TopSys of variable-basis lattice-valued topological systems of J. T. Denniston, A. Melton, and S. E. Rodabaugh [9].

To provide another example of categories of the form $\mathbf{A f} \mathbf{S y s}(T)$, an additional notion is needed.

Definition 10. A $T$-system $(X, \kappa, B)$ is called separated provided that $T X \stackrel{\kappa}{\rightarrow} B$ is an epimorphism in $\mathbf{B}^{o p}$. $\operatorname{AfSys}_{s}(T)$ is the full subcategory of $\operatorname{Af} \operatorname{Sys}(T)$ of separated $T$-systems.

We recall from, e.g., [6, pp. $393-394$ ] that monomorphisms in every variety $\mathbf{B}$ are necessarily injective; given a monomorphism $B \stackrel{\varphi}{\rightarrow} B^{\prime}$, the set $K=\left\{\left(b_{1}, b_{2}\right) \in B \times B \mid \varphi\left(b_{1}\right)=\varphi\left(b_{2}\right)\right\}$ is a subalgebra of $B \times B$ such that the respective projections $K \underset{\pi_{2}}{\stackrel{\pi_{1}}{\longrightarrow}} B$ satisfy $\varphi \circ \pi_{1}=\varphi \circ \pi_{2}$, and therefore, $\pi_{1}=\pi_{2}$.

Example 11. If $\mathbf{B}=\mathbf{C S L}$, then $\mathbf{A f} \operatorname{Sys}_{s}\left(\mathcal{P}_{2}\right)$ is the category $\mathbf{S P}$ of state property systems of D. Aerts $[4]$. 


\subsection{Affine spatialization procedure}

Following the results of [4], this subsection shows an affine analogue of the topological system spatialization procedure of S. Vickers.

\section{Theorem 12.}

(1) There is a full embedding $\operatorname{Af} \mathbf{S p c}(T) \stackrel{E}{\longrightarrow} \operatorname{AfSys}(T), \quad E\left(\left(X_{1}, \tau_{1}\right) \stackrel{f}{\rightarrow}\left(X_{2}, \tau_{2}\right)\right)=\left(X_{1}, e_{\tau_{1}}^{o p}, \tau_{1}\right) \stackrel{(f, \varphi)}{\longrightarrow}$ $\left(X_{2}, e_{\tau_{2}}^{o p}, \tau_{2}\right)$, where $e_{\tau_{i}}$ is the inclusion $\tau_{i} \hookrightarrow T X_{i}$, and $\varphi^{o p}$ is the restriction $\tau_{2} \stackrel{(T f)^{o p} \mid \tau_{2}^{\tau_{1}}}{\longrightarrow} \tau_{1}$.

(2) E has a right-adjoint-left-inverse $\mathbf{A f} \operatorname{Sys}(T) \stackrel{\text { Spat }}{\longrightarrow} \operatorname{Af} \operatorname{Spc}(T), \operatorname{Spat}\left(\left(X_{1}, \kappa_{1}, B_{1}\right) \stackrel{(f, \varphi)}{\longrightarrow}\left(X_{2}, \kappa_{2}, B_{2}\right)\right)=$ $\left(X_{1},\left(\kappa_{1}^{o p}\right) \rightarrow\left(B_{1}\right)\right) \stackrel{f}{\rightarrow}\left(X_{2},\left(\kappa_{2}^{o p}\right) \rightarrow\left(B_{2}\right)\right)$.

(3) $\operatorname{Af} \operatorname{Spc}(T)$ is isomorphic to a full (regular mono)-coreflective subcategory of $\operatorname{AfSys}(T)$.

Proof. To show that Spat is a right adjoint to $E$, it is enough to verify that every system $(X, \kappa, B)$ has an $E$-co-universal arrow, i.e., a $T$-morphism $\operatorname{ESpat}(X, \kappa, B) \stackrel{\varepsilon}{\rightarrow}(X, \kappa, B)$ such that for every $T$-morphism $E\left(X^{\prime}, \tau^{\prime}\right) \stackrel{(f, \varphi)}{\longrightarrow}(X, \kappa, B)$, there exists a unique $T$-morphism $\left(X^{\prime}, \tau^{\prime}\right) \stackrel{g}{\rightarrow} \operatorname{Spat}(X, \kappa, B)$ with $\varepsilon \circ E g=(f, \varphi)$.

There exists a $T$-morphism $\left(E S p a t(X, \kappa, B)=\left(X, e_{\left(\kappa^{o p}\right) \rightarrow(B)}^{o p},\left(\kappa^{o p}\right) \rightarrow(B)\right)\right) \stackrel{\varepsilon=\left(1_{X}, \kappa\right)}{\longrightarrow}(X, \kappa, B)$. Given a $T$-morphism $E\left(X^{\prime}, \tau^{\prime}\right) \stackrel{(f, \varphi)}{\longrightarrow}(X, \kappa, B)$, it follows that $(T f)^{o p} \circ \kappa^{o p}=e_{\tau^{\prime}} \circ \varphi^{o p}$, which yields the desired $T$-morphism $\left(X^{\prime}, \tau^{\prime}\right) \stackrel{f}{\rightarrow}\left(\operatorname{Spat}(X, \kappa, B)=\left(X,\left(\kappa^{o p}\right) \rightarrow(B)\right)\right)$, whose uniqueness is clear.

For the last claim, it is enough to show that given a $T$-system $(X, \kappa, B)$, the map $B \stackrel{\kappa^{o p}}{\longrightarrow}\left(\kappa^{o p}\right) \rightarrow(B)$ is a regular epimorphism in $\mathbf{B}$. Define $C=\left\{\left(b_{1}, b_{2}\right) \in B \times B \mid \kappa^{o p}\left(b_{1}\right)=\kappa^{o p}\left(b_{2}\right)\right\}$ (the kernel of $\kappa^{o p}$ ), and let $C \stackrel{\pi_{i}}{\longrightarrow} B$ be given by $\pi_{i}\left(b_{1}, b_{2}\right)=b_{i}$ for $i \in\{1,2\}$. Then $\left(\kappa^{o p},\left(\kappa^{o p}\right) \rightarrow(B)\right)$ is a coequalizer of $\left(\pi_{1}, \pi_{2}\right)$.

The analogue of Theorem 12 for the category $\operatorname{AfSys}_{s}(T)$ is even better.

Theorem 13. The functors $E$ and Spat restrict to $\mathbf{A f \operatorname { S p c }}(T) \stackrel{\bar{E}}{\longrightarrow} \operatorname{AfSys}_{s}(T)$ and $\mathbf{A f S y s}_{s}(T) \stackrel{\overline{\text { Spat }}}{\longrightarrow}$ $\operatorname{Af} \mathbf{S p c}(T)$, respectively, providing an equivalence between the categories $\mathbf{A f} \mathbf{S p c}(T)$ and $\mathbf{A f S y s}_{s}(T)$ such that $\overline{\operatorname{Spat}} \bar{E}=1_{\mathbf{A f S p c}(T)}$.

Proof. By Theorem 12, $\overline{\text { Spat }}$ is a right-adjoint-left-inverse to $\bar{E}$. To prove the theorem, it is enough to show that for every separated $T$-system $(X, \kappa, B)$, the $E$-co-universal arrow $\operatorname{ESpat}(X, \kappa, B) \stackrel{\varepsilon=\left(1_{X}, \kappa\right)}{\longrightarrow}(X, \kappa, B)$ from the proof of Theorem 12 is an isomorphism. The claim follows from the definition of $\varepsilon$, since $B \stackrel{\kappa^{o p}}{\longrightarrow}$ $\left(\kappa^{o p}\right) \rightarrow(B)$ is always surjective, and it is injective by the property of separated $T$-systems.

Corollary 14. The category $\mathbf{A f} \mathbf{S p c}(T)$ is the amnestic modification of the category $\mathbf{A f S y s}_{s}(T)$.

Proof. Follows from Theorem 13 and the definition of the amnestic modification of [2, Remark 5.34].

The following (well-known) results are direct consequences of Theorems 12 and 13, respectively.

\section{Remark 15.}

(1) Top is isomorphic to a full (regular mono)-coreflective subcategory of the category TopSys, which provides the system spatialization procedure of S. Vickers. More generally, Loc-Top (Loc is the dual of Frm) is isomorphic to a full (regular mono)-coreflective subcategory of the category Loc-TopSys.

(2) The categories $\mathbf{C l s}$ and $\mathbf{S P}$ are equivalent [3, 4].

Moreover, from Corollary 14, one gets [4, Theorem 4], which states that the category Cls is the amnestic modification of the category $\mathbf{S P}$. 


\section{Localification procedure for affine systems}

This section provides a localification procedure for affine systems, motivated by the above-mentioned localification procedure for topological systems of S. Vickers.

Proposition 16. There is a functor $\operatorname{AfSys}(T) \stackrel{\operatorname{Loc}}{\longrightarrow} \mathbf{B}^{o p}, \operatorname{Loc}\left(\left(X_{1}, \kappa_{1}, B_{1}\right) \stackrel{(f, \varphi)}{\longrightarrow}\left(X_{2}, \kappa_{2}, B_{2}\right)\right)=B_{1} \stackrel{\varphi}{\rightarrow} B_{2}$.

Unlike the affine spatialization procedure, in which the functor in the opposite direction always exists, the localification procedure is more demanding.

Theorem 17. Given a functor $\mathbf{X} \stackrel{T}{\rightarrow} \mathbf{B}^{o p}$, the following are equivalent.

(1) There exists an adjoint situation $(\eta, \varepsilon): T \dashv P t: \mathbf{B}^{o p} \rightarrow \mathbf{X}$.

(2) There exists a full embedding $\mathbf{B}^{o p} \stackrel{E}{\longrightarrow} \mathbf{A f} \mathbf{S y s}(T)$ such that Loc is a left-adjoint-left-inverse to $E$. $\mathbf{B}^{o p}$ is then isomorphic to a full reflective subcategory of $\mathbf{A f} \mathbf{S y s}(T)$.

Proof.

$A d(1) \Rightarrow(2)$. Define a functor $\mathbf{B}^{o p} \stackrel{E}{\rightarrow} \operatorname{AfSys}(T)$ by $E\left(B_{1} \stackrel{\varphi}{\rightarrow} B_{2}\right)=\left(P t B_{1}, \varepsilon_{B_{1}}, B_{1}\right) \stackrel{(P t \varphi, \varphi)}{\longrightarrow}$ $\left(P t B_{2}, \varepsilon_{B_{2}}, B_{2}\right)$. Correctness of $E$ on morphisms follows from commutativity of the diagram

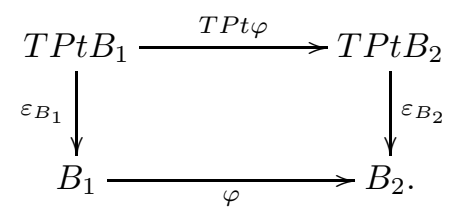

Moreover, $E$ is clearly an embedding. To verify that the functor $E$ is full, note that given a $T$-morphism $\left(P t B_{1}, \varepsilon_{B_{1}}, B_{1}\right) \stackrel{(f, \varphi)}{\longrightarrow}\left(P t B_{2}, \varepsilon_{B_{2}}, B_{2}\right)$, commutativity of the diagrams

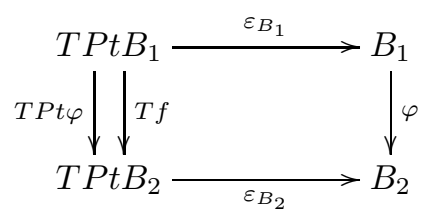

implies that $\varepsilon_{B_{2}} \circ T P t \varphi=\varepsilon_{B_{2}} \circ T f$, and therefore, $P t \varphi=f$. Given a $T$-system $(X, \kappa, B)$, straightforward calculations show that $(X, \kappa, B) \stackrel{\left(f:=P t \kappa \circ \eta_{X}, 1_{B}\right)}{\longrightarrow}\left(\left(P t B, \varepsilon_{B}, B\right)=E \operatorname{Loc}(X, \kappa, B)\right)$ provides an $E$-universal arrow for $(X, \kappa, B)$. It is also easy to see that $\operatorname{Loc} E=1_{\mathbf{B}^{o p}}$.

$A d(2) \Rightarrow(1)$. Given an adjunction $L o c \dashv E: \mathbf{B}^{o p} \rightarrow \operatorname{Af} \mathbf{S y s}(T), \mathbf{X} \stackrel{T}{\rightarrow} \mathbf{B}^{o p}$ is the composition of the left adjoint functors $\mathbf{X} \rightarrow \mathbf{A f} \mathbf{S p c}(T)$ (the indiscrete functor of, e.g., [2, Proposition 21.12 (2)], which exists by Theorem 17), $\operatorname{AfSpc}(T) \stackrel{E}{\longrightarrow} \operatorname{AfSys}(T)$ (the embedding of Theorem 12), and $\operatorname{AfSys}(T) \stackrel{L o c}{\longrightarrow} \mathbf{B}^{o p}$.

The following provides an example of the functor Pt of Theorem 17(1).

Theorem 18. Every functor Set $\stackrel{\mathcal{P}_{B}}{\longrightarrow} \mathbf{B}^{o p}$ has a right adjoint.

Proof. Recall that the right adjoint functor $\mathbf{B}^{o p} \stackrel{P t_{B}}{\longrightarrow}$ Set is given by $P t_{B}\left(B_{1} \stackrel{\varphi}{\rightarrow} B_{2}\right)=\mathbf{B}\left(B_{1}, B\right) \stackrel{P t_{B} \varphi}{\longrightarrow}$ $\mathbf{B}\left(B_{2}, B\right)$, where $\left(P t_{B} \varphi\right)(p)=p \circ \varphi^{o p}$. Given a B-algebra $A$, the map $A \stackrel{\varepsilon^{o p}}{\longrightarrow}\left(\mathcal{P}_{B} P t_{B} A=B^{\mathbf{B}(A, B)}\right)$, defined by $\left(\varepsilon^{o p}(a)\right)(p)=p(a)$, provides a $\mathcal{P}_{B}$-co-universal arrow for $A$.

As a consequence of Theorems 17, 18, one gets the following well-known results. 


\section{Remark 19.}

(1) Loc (the dual of Frm) is isomorphic to a full reflective subcategory of TopSys, which provides the system localification procedure of S. Vickers.

(2) $\mathbf{B}^{o p}$ is isomorphic to a full reflective subcategory of $\operatorname{Af} \operatorname{Sys}\left(\mathcal{P}_{B}\right)$.

In particular, the case of the category TopSys shows that in Theorem $17(2)$, the category $\mathbf{B}^{o p}$, even if a reflective subcategory of $\operatorname{Af} \operatorname{Sys}(T)$, can be both non-mono-reflexive and non-epi-reflexive.

In 38], S. E. Rodabaugh considered functors of the form Set $\times \mathbf{S} \stackrel{\mathcal{P}_{\mathbf{S}}}{\longrightarrow}$ Loc and their respective categories of affine spaces, using, however, a different terminology (recall Example 6(4)). The next result shows that Theorem 18 (in general) can not be extended from the subcategory $\left\{B \stackrel{1_{B}}{\longrightarrow} B\right\}$ to the whole $\mathbf{B}^{o p}$.

Proposition 20. Consider a functor Set $\times \mathbf{B}^{o p} \stackrel{T:=\mathcal{P}_{\mathbf{B}}^{o p}}{\longrightarrow} \mathbf{B}^{o p}$, and suppose that there exists a $\mathbf{B}$-algebra $B$, whose underlying set has finite cardinality $n>1$. Then $T$ has no right adjoint.

Proof. If $T$ has a right adjoint, then $T$ preserves coproducts. Given a singleton set $1, T((1, B) \bigsqcup(1, B))=$ $T((1 \biguplus 1, B \times B))=(B \times B)^{(1 \biguplus 1)}$ and $T(1, B) \times T(1, B)=B^{1} \times B^{1}$. Since $T((1, B) \coprod(1, B)) \cong T(1, B) \times$ $T(1, B), n^{4}=\operatorname{Card}\left((B \times B)^{(1 \biguplus 1)}\right)=\operatorname{Card}\left(B^{1} \times B^{1}\right)=n^{2}$, which is a contradiction.

For instance, Proposition 20 implies that the functor Set $\times$ Loc $\stackrel{\mathcal{P}_{\text {Loc }}}{\longrightarrow}$ Loc has no right adjoint, i.e., Theorem 17(2) is not applicable to the category Loc-TopSys of Example 9](3).

\subsection{Affine theories}

The results of this subsection stem from [45], and are motivated by the categorical approach to universal algebra, based on the concept of algebraic theory of F. W. Lawvere [33]. We would like to develop the tools, which would allow one to study the properties of a category of the form $\operatorname{AfSys}(T)$ (or $\operatorname{AfSpc}(T)$ ) through the properties of its related functor $\mathbf{X} \stackrel{T}{\rightarrow} \mathbf{B}^{o p}$.

Definition 21. An affine theory is a functor $\mathbf{X} \stackrel{T}{\rightarrow} \mathbf{B}^{o p}$, where $\mathbf{B}$ is a variety of algebras.

Throughout this paper, there is no distinction made between categories and quasicategories [2]. The latter are defined similarly to the former except that their objects do not necessarily form a class and their hom-families are not necessarily sets.

Definition 22. AfTh is the category, whose objects are affine theories $\mathbf{X} \stackrel{T}{\rightarrow} \mathbf{B}^{o p}$, and whose morphisms $T_{1} \stackrel{(F, \Phi, \eta)}{\longrightarrow} T_{2}$ (also shortened to $\eta$ ) comprise two functors $\mathbf{X}_{1} \stackrel{F}{\rightarrow} \mathbf{X}_{2}, \mathbf{B}_{1} \stackrel{\Phi}{\rightarrow} \mathbf{B}_{2}$ and a natural transformation $T_{2} F \stackrel{\eta}{\rightarrow} \Phi^{o p} T_{1}$, or, more specifically,

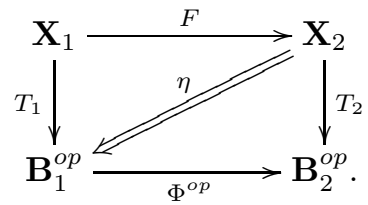

Given two affine theories $T_{1} \stackrel{\eta_{1}}{\longrightarrow} T_{2}$ and $T_{2} \stackrel{\eta_{2}}{\longrightarrow} T_{3}$, their composition is defined by $T_{3} F_{2} F_{1} \stackrel{\eta_{2} \odot \eta_{1}}{\longrightarrow} \Phi_{2}^{o p} \Phi_{1}^{o p} T_{1}=$ $T_{3} F_{2} F_{1} \stackrel{\eta_{2} F_{1}}{\longrightarrow} \Phi_{2}^{o p} T_{2} F_{1} \stackrel{\Phi_{2}^{o p} \eta_{1}}{\longrightarrow} \Phi_{2}^{o p} \Phi_{1}^{o p} T_{1}$. The identity on an affine theory $T$ is provided by the identity natural transformation $T \stackrel{1_{T}}{\longrightarrow} T$.

Note that the functor $\Phi^{o p}$ in Definition 22 goes in the same direction as $\Phi$, i.e., does not switch its domain and codomain. The next lemma shows that the construction of Definition 22 is indeed a category.

Lemma 23. The composition law is associative and the identities are as given. 
Proof. Consider three affine theory morphisms $T_{1} \stackrel{\eta_{1}}{\longrightarrow} T_{2}, T_{2} \stackrel{\eta_{2}}{\longrightarrow} T_{3}$ and $T_{3} \stackrel{\eta_{3}}{\longrightarrow} T_{4}$, or, more specifically, the following diagram:

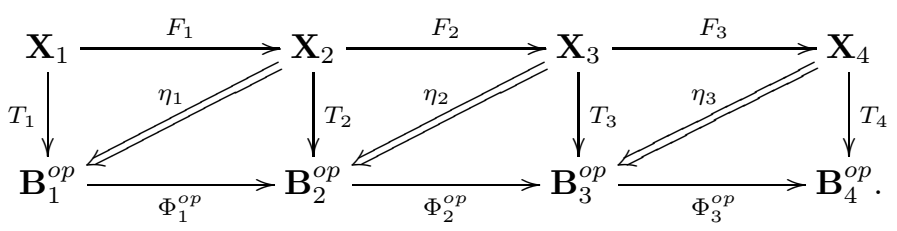

Straightforward computations show that

$$
\begin{array}{r}
T_{4} F_{3} F_{2} F_{1} \stackrel{\left(\eta_{3} \odot \eta_{2}\right) \odot \eta_{1}}{\longrightarrow} \Phi_{3}^{o p} \Phi_{2}^{o p} \Phi_{1}^{o p} T_{1}= \\
T_{4} F_{3} F_{2} F_{1} \stackrel{\left(\eta_{3} \odot \eta_{2}\right)_{F_{1}}}{\longrightarrow} \Phi_{3}^{o p} \Phi_{2}^{o p} T_{2} F_{1} \stackrel{\Phi_{3}^{o p} \Phi_{2}^{o p} \eta_{1}}{\longrightarrow} \Phi_{3}^{o p} \Phi_{2}^{o p} \Phi_{1}^{o p} T_{1}= \\
T_{4} F_{3} F_{2} F_{1} \stackrel{\eta_{3} F_{2} F_{1}}{\longrightarrow} \Phi_{3}^{o p} T_{3} F_{2} F_{1} \stackrel{\Phi_{3}^{o p} \eta_{2} F_{1}}{\longrightarrow} \Phi_{3}^{o p} \Phi_{2}^{o p} T_{2} F_{1} \stackrel{\Phi_{3}^{o p} \Phi_{2}^{o p} \eta_{1}}{\longrightarrow} \Phi_{3}^{o p} \Phi_{2}^{o p} \Phi_{1}^{o p} T_{1}= \\
T_{4} F_{3} F_{2} F_{1} \stackrel{\eta_{3}{ }_{F_{2} F_{1}}}{\longrightarrow} \Phi_{3}^{o p}\left(T_{3} F_{2} F_{1} \stackrel{\eta_{2} F_{1}}{\longrightarrow} \Phi_{2}^{o p} T_{2} F_{1} \stackrel{\Phi_{2}^{o p} \eta_{1}}{\longrightarrow} \Phi_{2}^{o p} \Phi_{1}^{o p} T_{1}\right)= \\
T_{4} F_{3} F_{2} F_{1} \stackrel{\eta_{3} F_{2} F_{1}}{\longrightarrow} \Phi_{3}^{o p} T_{3} F_{2} F_{1} \stackrel{\Phi_{3}^{o p}\left(\eta_{2} \odot \eta_{1}\right)}{\longrightarrow} \Phi_{3}^{o p} \Phi_{2}^{o p} \Phi_{1}^{o p} T_{1}= \\
T_{4} F_{3} F_{2} F_{1} \stackrel{\eta_{3} \odot\left(\eta_{2} \odot \eta_{1}\right)}{\longrightarrow} \Phi_{3}^{o p} \Phi_{2}^{o p} \Phi_{1}^{o p} T_{1}
\end{array}
$$

The last statement of the lemma is clear.

The composition law of the category AfTh resembles the star product of [29, Definition 13.10], but does not coincide with it.

Definition 24. AfStm is the category, whose objects are categories of the form $\operatorname{Af} \operatorname{Sys}(T)$ and whose morphisms are functors between them.

The following theorem shows that affine theory morphisms may be translated into functors between their respective categories of affine systems.

Theorem 25. There is a functor AfTh $\stackrel{\text { AfSys }}{\longrightarrow}$ AfStm, AfSys $\left(T_{1} \stackrel{\eta}{\rightarrow} T_{2}\right)=\operatorname{AfSys}\left(T_{1}\right) \stackrel{\text { AfSys }}{\longrightarrow} \operatorname{AfSys}\left(T_{2}\right)$, where AfSys $\left((X, \kappa, B) \stackrel{(f, \varphi)}{\longrightarrow}\left(X^{\prime}, \kappa^{\prime}, B^{\prime}\right)\right)=\left(F X, \Phi^{o p} \kappa \circ \eta_{X}, \Phi^{o p} B\right) \stackrel{\left(F f, \Phi^{o p} \varphi\right)}{\longrightarrow}\left(F X^{\prime}, \Phi^{o p} \kappa^{\prime} \circ \eta_{X^{\prime}}, \Phi^{o p} B^{\prime}\right)$.

Proof. We begin with the verification that the definition of AfSys $\eta$ is correct. Given an $\mathbf{A f} \operatorname{Sys}\left(T_{1}\right)$ morphism $(X, \kappa, B) \stackrel{(f, \varphi)}{\longrightarrow}\left(X^{\prime}, \kappa^{\prime}, B^{\prime}\right)$, the diagram

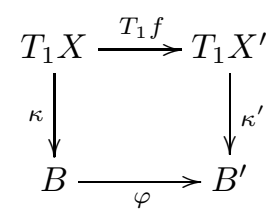

commutes and that provides the commutativity of the next one:

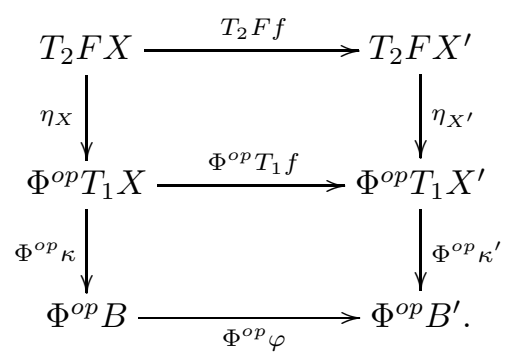


It follows that $\left(F X, \Phi^{o p} \kappa \circ \eta_{X}, \Phi^{o p} B\right) \stackrel{\left(F f, \Phi^{o p} \varphi\right)}{\longrightarrow}\left(F X^{\prime}, \Phi^{o p} \kappa^{\prime} \circ \eta_{X^{\prime}}, \Phi^{o p} B^{\prime}\right)$ is an Af Sys $\left(T_{2}\right)$-morphism.

To show that AfSys also preserves the composition, we notice that given two affine theory morphisms $T_{1} \stackrel{\left(F_{1}, \Phi_{1}, \eta_{1}\right)}{\longrightarrow} T_{2}$ and $T_{2} \stackrel{\left(F_{2}, \Phi_{2}, \eta_{2}\right)}{\longrightarrow} T_{3}$, it follows that

$$
\begin{gathered}
\operatorname{AfSys}\left(\left(F_{2}, \Phi_{2}, \eta_{2}\right) \circ\left(F_{1}, \Phi_{1}, \eta_{1}\right)\right)\left((X, \kappa, B) \stackrel{(f, \varphi)}{\longrightarrow}\left(X^{\prime}, \kappa^{\prime}, B^{\prime}\right)\right)= \\
\left.\operatorname{AfSys}\left(F_{2} F_{1}, \Phi_{2} \Phi_{1}, \eta_{2} \odot \eta_{1}\right)\left((X, \kappa, B) \stackrel{(f, \varphi)}{\longrightarrow}\left(X^{\prime}, \kappa^{\prime}, B^{\prime}\right)\right)\right)= \\
\left(F_{2} F_{1} X, \Phi_{2}^{o p} \Phi_{1}^{o p} \kappa \circ\left(\eta_{2} \odot \eta_{1}\right)_{X}, \Phi_{2}^{o p} \Phi_{1}^{o p} B,\right) \stackrel{\left(F_{2} F_{1} f, \Phi_{2}^{o p} \Phi_{1}^{o p} \varphi\right)}{\longrightarrow} \\
\left(F_{2} F_{1} X^{\prime}, \Phi_{2}^{o p} \Phi_{1}^{o p} \kappa^{\prime} \circ\left(\eta_{2} \odot \eta_{1}\right)_{X^{\prime}}, \Phi_{2}^{o p} \Phi_{1}^{o p} B^{\prime}\right) \stackrel{(\dagger)}{=} \\
\left(F_{2} F_{1} X, \Phi_{2}^{o p}\left(\Phi_{1}^{o p} \kappa \circ \eta_{1 X}\right) \circ \eta_{2} F_{1} X, \Phi_{2}^{o p} \Phi_{1}^{o p} B\right) \stackrel{\left(F_{2} F_{1} f, \Phi_{2}^{o p} \Phi_{1}^{o p} \varphi\right)}{\longrightarrow} \\
\left(F_{2} F_{1} X^{\prime}, \Phi_{2}^{o p}\left(\Phi_{1}^{o p} \kappa^{\prime} \circ \eta_{1 X^{\prime}}\right) \circ \eta_{2} F_{1} X^{\prime}, \Phi_{2}^{o p} \Phi_{1}^{o p} B^{\prime}\right)= \\
\text { AfSys }\left(F_{2}, \Phi_{2}, \eta_{2}\right)\left(\left(F_{1} X, \Phi_{1}^{o p} \kappa \circ \eta_{1 X}, \Phi_{1}^{o p} B\right) \stackrel{\left(F_{1} f, \Phi_{1}^{o p} \varphi\right)}{\longrightarrow}\right. \\
\left.\left(F_{1} X^{\prime}, \Phi_{1}^{o p} \kappa^{\prime} \circ \eta_{1} X^{\prime}, \Phi_{1}^{o p} B^{\prime}\right)\right)= \\
\text { AfSys }\left(F_{2}, \Phi_{2}, \eta_{2}\right) \circ \text { AfSys }\left(F_{1}, \Phi_{1}, \eta_{1}\right)\left((X, \kappa, B) \stackrel{(f, \varphi)}{\longrightarrow}\left(X^{\prime}, \kappa^{\prime}, B^{\prime}\right)\right),
\end{gathered}
$$

where $(\dagger)$ relies on the fact that $\Phi_{2}^{o p} \Phi_{1}^{o p} \kappa \circ\left(\eta_{2} \odot \eta_{1}\right)_{X}=\Phi_{2}^{o p} \Phi_{1}^{o p} \kappa \circ \Phi_{2}^{o p} \eta_{1 X} \circ \eta_{2} F_{1} X=\Phi_{2}^{o p}\left(\Phi_{1}^{o p} \kappa \circ \eta_{1 X}\right) \circ \eta_{2} F_{1} X$.

Preservation of the identities is straightforward.

It is an interesting and challenging open question, whether the functor of Theorem 25] has a left or right adjoint.

For the sake of completeness, it is noted that by analogy with the category AfStm, one can define the category, which comprises the categories of the form $\mathbf{A f} \mathbf{S p c}(T)$. The respective functor from $\mathbf{A f T h}$ in this case though requires more effort and will be not considered here; this topic is studied in full detail in [45].

\section{Affine systems as a framework for elementary institutions}

In this section is proposed a new framework for doing a certain type of institutions, namely, elementary institutions. This new framework is based in affine systems.

\subsection{Institutions and their morphisms}

The definitions of institution and its morphism are recalled from, e.g., [26] (elementary institutions or also pre-institutions come from [40, 42]). From now on, Cat denotes the category of categories (recall there is no distinction made between categories and quasicategories).

Definition 26. An institution $\mathbb{I}$ consists of:

- a category Sign of signatures with $\Sigma$ denoting an arbitrary object,

- a functor Sign $\stackrel{M o d}{\longrightarrow}$ Cat $^{o p}$ giving $\Sigma$-models and $\Sigma$-morphisms,

- a functor Sign $\stackrel{S e n}{\longrightarrow}$ Set giving $\Sigma$-sentences,

- a satisfaction relation $\models_{\Sigma} \subseteq O b(\operatorname{Mod} \Sigma) \times \operatorname{Sen} \Sigma$ for every $\Sigma \in O b($ Sign $)$

such that for every Sgn-morphism $\Sigma \stackrel{\phi}{\rightarrow} \Sigma^{\prime}$, the Satisfaction Condition

$$
m^{\prime} \models_{\Sigma^{\prime}} \operatorname{Sen} \phi(s) \text { iff } \operatorname{Mod} \phi\left(m^{\prime}\right) \models_{\Sigma} s
$$

holds for every $m^{\prime} \in \operatorname{Ob}\left(\operatorname{Mod} \Sigma^{\prime}\right)$ and every $s \in \operatorname{Sen} \Sigma$. An institution is called elementary [41, Definition 3.1], [42] (also pre-institution [40, Definition 1]) provided that the category Cat (in the definition of the functor Mod) is replaced with the category Set. 
Elementary institutions are not much popular among the researchers at the moment. There do exist though several applications of this concept, which could be found in, e.g., 17, 40, 41, 42.

We notice that [25] defines elementary institutions under the name of simplest institution. There also exists a more sophisticated concept of elementary institution of [13, p. 68], which is not used in this paper.

The above satisfaction relation $\models_{\Sigma} \subseteq \operatorname{Ob}(\operatorname{Mod} \Sigma) \times \operatorname{Sen} \Sigma$ can be rewritten as a map $\operatorname{Ob}(\operatorname{Mod} \Sigma) \stackrel{\kappa}{\rightarrow}$ $\mathcal{P}(\operatorname{Sen} \Sigma)$, in which $\kappa(m)=\left\{e \in \operatorname{Sen} \Sigma \mid m \models_{\Sigma} e\right\}$. Moreover, if one defines 2 as the category $\perp \stackrel{\iota}{\rightarrow} \top$ (two objects and one non-identity morphism), every functor $\operatorname{Mod} \Sigma \stackrel{K}{\longrightarrow} \mathbf{2}^{\operatorname{Sen} \Sigma}$ will provide a satisfaction relation $\models_{\Sigma} \subseteq \operatorname{Ob}(\operatorname{Mod} \Sigma) \times \operatorname{Sen} \Sigma$ with $m \models_{\Sigma} e$ iff $K m(e)=\top$.

Definition 27. Let $\mathbb{I}$ and $\mathbb{I}^{\prime}$ be institutions. Then an institution morphism $\mathbb{I} \stackrel{(\Phi, \alpha, \beta)}{\longrightarrow} \mathbb{I}^{\prime}$ consists of:

- a functor Sign $\stackrel{\Phi}{\rightarrow}$ Sign', $^{\prime}$

- a natural transformation $\operatorname{Sen}^{\prime} \Phi \stackrel{\alpha}{\rightarrow}$ Sen, or, more specifically,

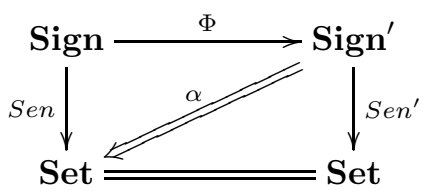

- a natural transformation $M o d \stackrel{\beta}{\rightarrow} M o d^{\prime} \Phi$ or, more specifically,

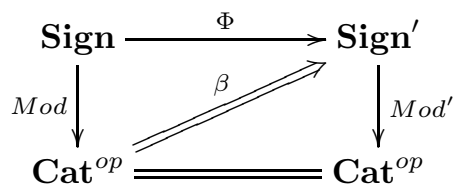

such that the following satisfaction condition holds

$$
m \models_{\Sigma} \alpha_{\Sigma}\left(s^{\prime}\right) \text { iff } \beta_{\Sigma}(m) \models_{\Phi \Sigma}^{\prime} s^{\prime}
$$

for every $\Sigma$-model $m$ from $\mathbb{I}$ and every $\Phi \Sigma$-sentence $s^{\prime}$ from $\mathbb{I}^{\prime}$.

Definitions 26, 27 provide the category Inst (resp., ElInst) of (resp., elementary) institutions and their morphisms. Examples of institutions include, e.g., first-order logic (with equality), Horn clause logic (with equality), equational logic, order-sorted equational logic, continuous equational logic [25] (we again recommend the reader to have a look at the more comprehensive list of examples of [13, Subsection 3.2]).

Following [26], the direction of natural transformations $\alpha, \beta$ in Definition 27 is (in a certain sense) arbitrary. More precisely, having natural transformations $\operatorname{Sen} \stackrel{\alpha}{\rightarrow} \operatorname{Sen}^{\prime} \Phi$ and $\operatorname{Mod}^{\prime} \Phi \stackrel{\beta}{\rightarrow} \operatorname{Mod}$ in hand, one gets the satisfaction condition

$$
\beta_{\Sigma}\left(m^{\prime}\right) \models_{\Sigma} s \text { iff } m^{\prime} \models_{\Phi \Sigma}^{\prime} \alpha_{\Sigma}(s)
$$

for every $\Sigma$-sentence $s$ from $\mathbb{I}$ and every $\Phi \Sigma$-model $m^{\prime}$ from $\mathbb{I}^{\prime}$. Such a structure is called institution comorphism [23]. We notice that comorphisms are more used than morphisms in institution theory. Moreover, the duality between morphisms and comorphisms is explained in [13.

\subsection{Topological institutions and their morphisms}

Following [42], we recall the notion of topological institution, which is based in the category TopSys of topological systems of S. Vickers (recall Example 9(1)).

Definition 28. A topological institution consists of: 
- a category Sign (of signatures),

- a functor Sign $\stackrel{\mathcal{T}}{\rightarrow} \operatorname{TopSys}^{o p}$.

Definition 29. A topological institution morphism $(\mathbf{S i g n}, \mathcal{T}) \stackrel{(\Phi, \alpha)}{\longrightarrow}\left(\mathbf{S i g n}^{\prime}, \mathcal{T}^{\prime}\right)$ consists of:

- a functor Sign $\stackrel{\Phi}{\rightarrow}$ Sign' $^{\prime}$

- a natural transformation $\mathcal{T} \stackrel{\alpha}{\rightarrow} \mathcal{T}^{\prime} \Phi$.

Definitions 28, 29 provide the category TpInst of topological institutions and their morphisms. Moreover, in [41, 42] an adjoint situation between the categories ElInst and TpInst is constructed. For convenience of the reader, we provide a brief description of some of its details.

We recall first from [49] that a topological system is a triple $(X, A, \models)(\operatorname{denoted} D=(\operatorname{pt} D, \Omega D, \models))$, where $X$ is a set, $A$ is a locale, and $\models \subseteq X \times A$ is a binary relation such that

(1) if $S$ is a finite subset of $A$, then for every $x \in X, x \models \bigwedge A$ iff $x \models a$ for every $a \in A$;

(2) if $S$ is any subset of $A$, then for every $x \in X, x \models \bigvee A$ iff $x \models a$ for some $a \in A$.

A topological system morphism $D_{1} \stackrel{f=(\mathrm{pt} f, \Omega f)}{\longrightarrow} D_{2}$ consists of a map pt $D_{1} \stackrel{\text { pt } f}{\longrightarrow}$ pt $D_{2}$ and a homomorphism of locales $\Omega D_{1} \stackrel{\Omega f}{\longrightarrow} \Omega D_{2}$ such that for every $x_{1} \in \operatorname{pt} D_{1}, a_{2} \in \Omega D_{2}, x_{1} \models_{1}(\Omega f)^{o p}\left(a_{2}\right)$ iff pt $f\left(x_{1}\right) \models_{2} a_{2}$.

We continue with some necessary institutional preliminaries from [41, 42]. Let (Sign, Mod, Sen, $\models$ ) be an elementary institution, and let $\Sigma \in O b$ (Sign) be an abstract signature. If $\Phi \subseteq \operatorname{Sen} \Sigma$ and $\psi \in \operatorname{Sen} \Sigma$, then $\Phi$ semantically entails $\psi$ (denoted $\Phi \vdash_{\Sigma} \psi$ ) provided that for every $x \in \operatorname{Mod} \Sigma, x \models_{\Sigma} \varphi$ for every $\varphi \in \Phi$, implies $x \models_{\Sigma} \psi$. One denotes $\Phi^{\vdash_{\Sigma}}=\left\{\psi \in \operatorname{Sen} \Sigma \mid \Phi \vdash_{\Sigma} \psi\right\}$. $\Phi$ is then called a theory provided that $\Phi^{\vdash_{\Sigma}}=\Phi$. The class of all theories over $\Sigma$ is denoted $\left|T h_{\Sigma}\right|$. On the class $\left|T h_{\Sigma}\right|$, one introduces a partial order by $\Phi_{1} \leqslant_{\Sigma} \Phi_{2}$ iff $\Phi_{1} \vdash_{\Sigma} \Phi_{2}$ (in the sense of the above semantical entailment relation $\vdash_{\Sigma}$ ) and obtains a complete lattice $T h_{\Sigma}^{o p}=\left(\left|T h_{\Sigma}\right|, \leqslant_{\Sigma}\right)$, in which, given $S \subseteq\left|T h_{\Sigma}\right|, \bigvee S=\bigcap S$ and $\bigwedge S=(\bigcup S)^{\vdash \Sigma}$. We get thus a triple $\left(\operatorname{Mod} \Sigma, T h_{\Sigma}^{o p}, \Vdash_{\Sigma}\right)$, in which $x \Vdash_{\Sigma} \Phi$ means $x \models_{\Sigma} \varphi$ for every $\varphi \in \Phi$. One can use now the coverage technique of [32] to complete the $\Lambda$-semilattice of theories, while preserving only the joins, which are respected by the satisfaction relation. Afterwards one extends the satisfaction relation to the new theories. In such a way, one obtains a functor ElInst $\stackrel{T o p}{\longrightarrow}$ TpInst (we omit its definition on morphisms).

The functor in the opposite direction TpInst $\stackrel{\text { Geo }}{\longrightarrow}$ ElInst is easier. Given a topological institution $\mathcal{T}$, one obtains an elementary institution (Sign, $\operatorname{Mod}$, Sen, $\models$ ), in which $\operatorname{Mod} \Sigma=\operatorname{pt} \mathcal{T} \Sigma, \operatorname{Sen} \Sigma=|\Omega \mathcal{T} \Sigma|$ (where $|-|$ stands for the underlying set), and $\models_{\Sigma}=\models_{\mathcal{T} \Sigma}$. One can show that Geo is a left adjoint to Top (41, Theorem 4.44],[42, Theorem 34]). It is not clear to us at the moment, whether one could provide a generalization of the just mentioned machinery for the category Inst of institutions.

\subsection{Affine institutions and their morphisms}

Using the ideas of the previous subsection, the concepts of affine institution and affine institution morphism are now introduced.

Definition 30. An affine institution consists of:

- a category $\mathbf{S}$ (of abstract signatures),

- an affine theory $T$,

- a functor $\mathbf{S} \stackrel{I}{\rightarrow} \operatorname{Af} \operatorname{Sys}(T)$.

Following the remark of the referee, we notice that unlike the case of topological institutions, affine institutions have their category of signatures denoted $\mathbf{S}$. The only reason for the change is our wish to, first, shorten the notations related to affine institutions, and, second, underline the abstract nature of signatures. 
Definition 31. An affine institution morphism $\left(\mathbf{S}_{1}, T_{1}, I_{1}\right) \stackrel{(\Phi, \eta, \alpha)}{\longrightarrow}\left(\mathbf{S}_{2}, T_{2}, I_{2}\right)$ consists of:

- a functor $\mathbf{S}_{1} \stackrel{\Phi}{\rightarrow} \mathbf{S}_{2}$

- an affine theory morphism $T_{1} \stackrel{\eta}{\rightarrow} T_{2}$,

- a natural transformation AfSys $I_{1} \stackrel{\alpha}{\rightarrow} I_{2} \Phi$ (recall the notation of Theorem 25), or, more specifically,

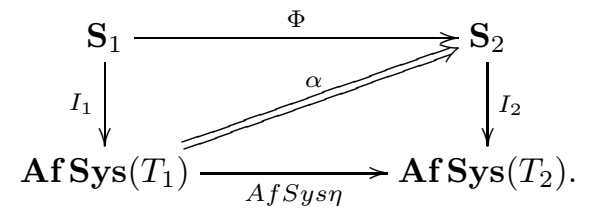

Definitions 30, 31 provide the category AfInst of affine institutions and their morphisms. For example, given affine institution morphisms

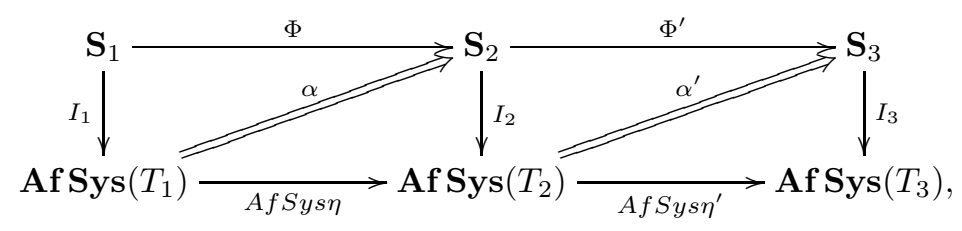

their respective composition is defined by AfSys $\left(\eta^{\prime} \circ \eta\right) I_{1} \stackrel{\alpha^{\prime} \boxminus \alpha}{\longrightarrow} I_{3} \Phi^{\prime} \Phi=$ AfSys $\eta^{\prime}$ AfSys $I_{1} \stackrel{\text { AfSys } \eta^{\prime} \alpha}{\longrightarrow}$ AfSys $\eta^{\prime} I_{2} \Phi \stackrel{\alpha^{\prime} \Phi}{\longrightarrow} I_{3} \Phi^{\prime}$ (cf. Definition 22 and Lemma 23).

Following the remarks after Definition 27, the direction of the natural transformation $\alpha$ in affine institution morphisms (cf. Definition 29) is changed.

Definition 32. Given an affine theory $T, \operatorname{AfInst}(T)$ is the subcategory of AfInst consisting of affine institutions $(\mathbf{S}, T, I)$ (shortened to $(\mathbf{S}, I))$ and their respective morphisms $\left(\Phi, 1_{T}, \alpha\right)$ (shortened to $(\Phi, \alpha)$ ).

\section{Example 33.}

(1) If $\mathbf{B}=\mathbf{F r m}$, then the category $\operatorname{AfInst}\left(\mathcal{P}_{2}\right)$ provides a modification of the category $\mathbf{T p I n s t}$ of [42].

(2) The affine theory Set $\stackrel{|\mathcal{P}|}{\longrightarrow}$ Set $^{o p}:=$ Set $\stackrel{\mathcal{P}}{\longrightarrow} \mathbf{C B A l g}^{o p} \stackrel{|-|^{o p}}{\longrightarrow}$ Set $^{o p}$, in which $|-|$ is the obvious forgetful functor, gives the category $\operatorname{AfInst}(|\mathcal{P}|)$, which is a modification of the category ElInst (recall the remark at the end of Definition 26].

\subsection{Affine institution spatialization and localification procedures}

In this subsection, a possible approach to spatialization and localification procedures for affine institutions is presented. Following the ideas of [41, 42], we begin with some preliminary definitions.

Definition 34. Let $T$ be an affine theory. A spatial affine $T$-institution consists of:

- a category $\mathbf{S}$ (of abstract signatures),

- a functor $\mathbf{S} \stackrel{I}{\rightarrow} \operatorname{Af} \operatorname{Spc}(T)$.

A spatial affine $T$-institution morphism $\left(\mathbf{S}_{1}, I_{1}\right) \stackrel{(\Phi, \alpha)}{\longrightarrow}\left(\mathbf{S}_{2}, I_{2}\right)$ consists of:

- a functor $\mathbf{S}_{1} \stackrel{\Phi}{\rightarrow} \mathbf{S}_{2}$, 
- a natural transformation $I_{1} \stackrel{\alpha}{\rightarrow} I_{2} \Phi$.

$\operatorname{SAfInst}(T)$ is the category of spatial affine $T$-institutions and their morphisms.

Definition 35. Let $\mathbf{X} \stackrel{T}{\rightarrow} \mathbf{B}^{o p}$ be an affine theory. A localic affine $T$-institution consists of:

- a category $\mathbf{S}$ (of abstract signatures),

- a functor $\mathbf{S} \stackrel{I}{\rightarrow} \mathbf{B}^{o p}$.

A localic affine $T$-institution morphism $\left(\mathbf{S}_{1}, I_{1}\right) \stackrel{(\Phi, \alpha)}{\longrightarrow}\left(\mathbf{S}_{2}, I_{2}\right)$ consists of:

- a functor $\mathbf{S}_{1} \stackrel{\Phi}{\rightarrow} \mathbf{S}_{2}$,

- a natural transformation $I_{1} \stackrel{\alpha}{\rightarrow} I_{2} \Phi$.

LAfInst $(T)$ is the category of localic affine $T$-institutions and their morphisms.

Example 36. If $\mathbf{B}=\mathbf{F r m}$, then the categories $\operatorname{SAfInst}\left(\mathcal{P}_{2}\right)$ and $\mathbf{L A f I n s t}\left(\mathcal{P}_{2}\right)$ provide (slightly) modified versions of the categories of spatial and localic topological institution of [41, 42], respectively.

From Theorems 12 and 17 (employing their respective notations), the following results are obtained.

\section{Theorem 37.}

(1) There exists a full embedding $\mathbf{S A f I n s t}(T) \stackrel{I E}{\longrightarrow} \operatorname{AfInst}(T)$ given by $I E\left(\left(\mathbf{S}_{1}, I_{1}\right) \stackrel{(\Phi, \alpha)}{\longrightarrow}\left(\mathbf{S}_{2}, I_{2}\right)\right)=$ $\left(\mathbf{S}_{1}, E I_{1}\right) \stackrel{(\Phi, E \alpha)}{\longrightarrow}\left(\mathbf{S}_{2}, E I_{2}\right)$.

(2) IE has a right-adjoint-left-inverse $\mathbf{A f I n s t}(T) \stackrel{I \text { Spat }}{\longrightarrow} \operatorname{SAfInst}(T), \operatorname{ISpat}\left(\left(\mathbf{S}_{1}, I_{1}\right) \stackrel{(\Phi, \alpha)}{\longrightarrow}\left(\mathbf{S}_{2}, I_{2}\right)\right)=$ $\left(\mathbf{S}_{1}\right.$, Spat $\left._{1}\right) \stackrel{(\Phi, \text { Spat })}{\longrightarrow}\left(\mathbf{S}_{2}\right.$, Spat $\left._{2}\right)$.

(3) $\mathbf{S A f I n s t}(T)$ is isomorphic to a full coreflective subcategory of $\mathbf{A f I n s t}(T)$.

Proof. For convenience of the reader, a sketch of the proof is given. Theorem[12 supplies us with an adjoint situation $(\eta, \varepsilon): E \dashv \operatorname{Spat}: \operatorname{Af} \mathbf{S y s}(T) \rightarrow \operatorname{Af} \mathbf{S p c}(T)$, in which $1_{\mathbf{A f S p c}(T)} \stackrel{\eta}{\rightarrow}$ SpatE and $E S p a t \stackrel{\varepsilon}{\rightarrow} 1_{\mathbf{A f S y s}(T)}$ are natural transformations such that Spate $\circ \eta$ Spat $=1_{S p a t}$ and $\varepsilon E \circ E \eta=1_{E}$.

Starting from $1_{\mathbf{A f S p c}(T)} \stackrel{\eta}{\rightarrow}$ SpatE, one obtains a natural transformation $1_{\mathbf{S A f I n s t}(T)} \stackrel{\Theta}{\rightarrow} \operatorname{ISpatIE}$, which is given for every spatial affine $T$-institution $(\mathbf{S}, I)$ by the following diagram

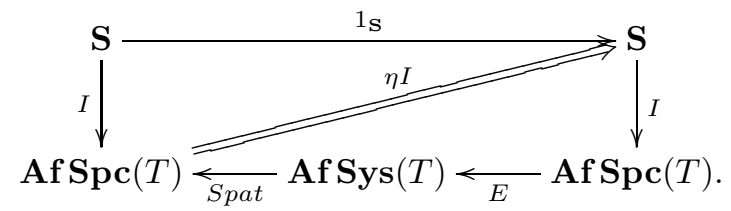

To see that $\Theta$ is indeed a natural transformation, it needs to be shown that given a spatial affine $T$-institution morphism $\left(\mathbf{S}_{1}, I_{1}\right) \stackrel{(\Phi, \alpha)}{\longrightarrow}\left(\mathbf{S}_{2}, I_{2}\right)$, the diagram

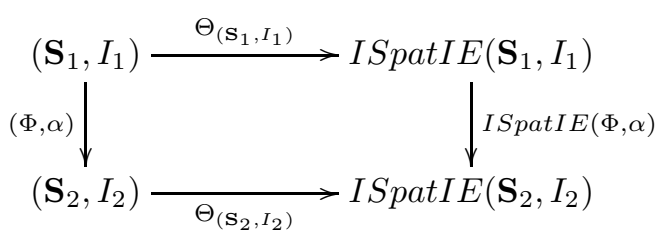


commutes. However, the commutivity of the previous diagram follows because the next diagram

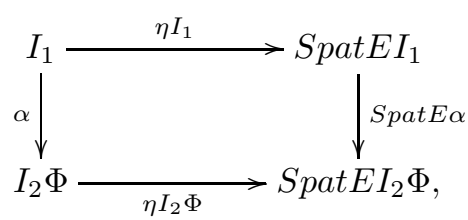

is clearly commutative ( $\eta$ is a natural transformation).

In a similar way, one gets the natural transformation IEISpat $\stackrel{\Upsilon}{\rightarrow} 1_{\text {AfInst }(T)}$. Moreover, to show commutativity of the triangle

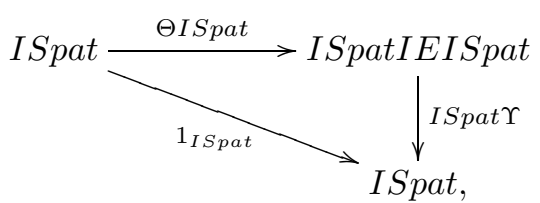

one notices that ISpat $\Upsilon \odot \Theta$ Spat $=1_{\text {ISpat }}$ is nothing else than Spate $\circ \eta$ Spat $=1_{\text {Spat }}$. Analogously, one arrives at $\Upsilon I E \circ I E \Theta=1_{I E}$, which concludes the proof.

Theorem 38. Let $T$ be an affine theory such that there exists an adjoint situation $(\eta, \varepsilon): T \dashv P t: \mathbf{B}^{o p} \rightarrow \mathbf{X}$.

(1) There exists a functor $\mathbf{A f I n s t}(T) \stackrel{I L o c}{\longrightarrow} \operatorname{LAfInst}(T)$, which is defined by $I \operatorname{Loc}\left(\left(\mathbf{S}_{1}, I_{1}\right) \stackrel{(\Phi, \alpha)}{\longrightarrow}\left(\mathbf{S}_{2}, I_{2}\right)\right)=$ $\left(\mathbf{S}_{1}, \operatorname{LocI}_{1}\right) \stackrel{(\Phi, L o c \alpha)}{\longrightarrow}\left(\mathbf{S}_{2}, \operatorname{LocI}_{2}\right)$.

(2) There exists a full embedding $\mathbf{L A f I n s t}(T) \stackrel{I E}{\longrightarrow} \operatorname{AfInst}(T)$ given by $\operatorname{IE}\left(\left(\mathbf{S}_{1}, I_{1}\right) \stackrel{(\Phi, \alpha)}{\longrightarrow}\left(\mathbf{S}_{2}, I_{2}\right)\right)=$ $\left(\mathbf{S}_{1}, E I_{1}\right) \stackrel{(\Phi, E \alpha)}{\longrightarrow}\left(\mathbf{S}_{2}, E I_{2}\right)$ such that ILoc is a left-adjoint-left-inverse to IE.

(3) $\mathbf{L A f I n s t}(T)$ is isomorphic to a full reflective subcategory of $\mathbf{A f I n s t}(T)$.

Proof. The proof is similar to that of Theorem 37, relying on Theorem 17 instead of Theorem 12

Theorem 37 gives an answer to the question regarding a spatialization construction for topological institutions posed in [42, p. 424] (the authors though have refrained from establishing the respective coreflection).

\section{Conclusion and future work}

Following the concept of topological institution of [41, 42], we introduced the notion of affine institution and showed its respective spatialization and localification procedures. Affine institutions seem to provide a better framework for elementary institutions and topological institutions because they do not require that the employed algebraic structures have to be frames. More precisely, while the authors of 41, 42] impose the frame structure on the set of theories (certain "closed" subsets of the set of sentences) of a given signature, which results in certain technical difficulties, we suggest the use of an arbitrary algebraic structure, which could be determined in each concrete case. Additionally, it is important to note that the idea of topological system, expressed in terms of affine systems, provides a convenient framework for, and is more general than, elementary institutions. This conclusion is in striking difference with the result of J. T. Denniston, A. Melton and S. E. Rodabaugh [11], which eventually represents lattice-valued topological systems as special lattice-valued institutions.

We end this paper with four open problems. 


\subsection{Institutions based in categories}

This paper considered a simplification of the concept of institution, which relies on the category Set of sets instead of the category Cat of categories. To incorporate the case of general institutions and their morphisms of Definitions 26, 27, one could introduce the following concepts.

Definition 39. Given a functor $\mathbf{C a t} \stackrel{T}{\rightarrow} \mathbf{C a t}^{o p}, \operatorname{GAfSys}(T)$ is the comma category $\left(T \downarrow 1_{\mathbf{C a t}^{o p}}\right)$, concrete over the product category $\mathbf{C a t} \times \mathbf{C} \mathbf{C a t}^{o p}$, whose objects (generalized $T$-affine systems) are triples $(\mathbf{X}, K, \mathbf{B})$, which are made by $\mathbf{C a t}^{o p}$-morphisms $T \mathbf{X} \stackrel{K}{\longrightarrow} \mathbf{B}$; and whose morphisms (generalized T-affine system morphisms $)\left(\mathbf{X}_{1}, K_{1}, \mathbf{B}_{1}\right) \stackrel{(F, \Phi)}{\longrightarrow}\left(\mathbf{X}_{2}, K_{2}, \mathbf{B}_{2}\right)$ are $\mathbf{C a t} \times \mathbf{C a t}^{o p}$-morphisms $\left(\mathbf{X}_{1}, \mathbf{B}_{1}\right) \stackrel{(F, \Phi)}{\longrightarrow}\left(\mathbf{X}_{2}, \mathbf{B}_{2}\right)$, making the diagram

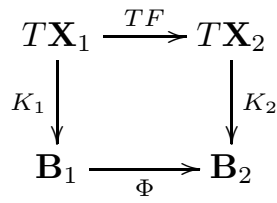

commute.

For convenience, a functor $\mathbf{C a t} \stackrel{T}{\rightarrow} \mathbf{C a t}^{o p}$ is called a generalized affine theory.

Definition 40. A generalized affine institution consists of:

- a category $\mathbf{S}$ (of abstract signatures),

- a generalized affine theory $T$,

- a functor $\mathbf{S} \stackrel{I}{\rightarrow} \operatorname{GAfSys}(T)$.

Definition 41. A generalized affine institution morphism $\left(\mathbf{S}_{1}, T_{1}, I_{1}\right) \stackrel{(F, \Phi, \alpha)}{\longrightarrow}\left(\mathbf{S}_{2}, T_{2}, I_{2}\right)$ consists of:

- a functor $\mathbf{S}_{1} \stackrel{F}{\rightarrow} \mathbf{S}_{2}$,

- a functor $\operatorname{GAfSys}\left(T_{1}\right) \stackrel{\Phi}{\rightarrow} \operatorname{GAfSys}\left(T_{2}\right)$,

- a natural transformation $I_{2} F \stackrel{\alpha}{\rightarrow} \Phi I_{1}$, or, more specifically,

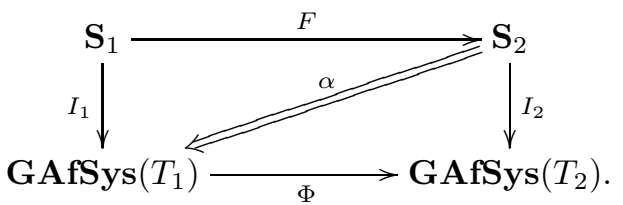

Definitions 40, 41 provide the category GAfInst of generalized affine institutions and their morphisms. The remarks after Definition 26 say that the category GAfInst is a generalization of the category Inst. The two next problems then arise immediately.

Problem 42. What are the spatialization and localification procedures for the category GAfInst?

Problem 43. What are the morphisms between two given generalized affine theories $T_{1}, T_{2}$, which could induce functors between the respective categories $\operatorname{GAfSys}\left(T_{1}\right), \operatorname{GAfSys}\left(T_{2}\right)$, so that one could use them (the morphisms) in the definition of the category GAfInst (cf. the definition of the category AfInst)? 


\subsection{Variable-basis spatialization and localification procedures for institutions}

Theorems 37 38 deal with the fixed-basis (in the sense of 31]) category $\operatorname{AfInst}(T)$ of affine institutions over a given affine theory $T$. One could be interested, however, in the whole variable-basis (in the sense of [38]) category AfInst.

Problem 44. What are the spatialization and localification procedures for the category AfInst?

Note that one still does not have a description of possible spatialization and localification procedures for affine spaces and systems, based in different affine theories. More precisely, Theorems 12, 17 are fixed-basis w.r.t. the employed affine theory $T$.

\subsection{Bornological systems and institutions}

Motivated by the concept of lattice-valued (fixed-basis) bornological space of [1], 37] introduced the notion of bornological vector system as a bornological analogue of topological systems of S. Vickers (see, e.g., 30] for an introduction into the theory of bornological spaces and their related concepts). In particular, the authors of [37] provided a spatialization procedure for bornological vector systems (see also [36]). Thus, the last open problem of this paper is as follows.

Problem 45. What are the relationships (if any) between bornological systems and institutions? What kind of institutions could arise from bornological systems?

We notice that the second part of Problem 45 could start a whole new development in the theory of institutions, providing, e.g., bornological institutions (à la topological institutions of [41, 42]).

The above-mentioned open problems will be addressed in our future papers on the topic of institutions.

\section{Acknowledgements}

The authors would like to express their sincere gratitude to the anonymous referees of this paper for their helpful remarks and suggestions on its possible improvements.

This is a preprint of the paper published in "Fuzzy Sets and Systems". The final authenticated version of the paper is available online at: https://www.sciencedirect.com/science/article/pii/S0165011415003668.

\section{References}

[1] M. Abel and A. Šostak, Towards the theory of L-bornological spaces, Iran. J. Fuzzy Syst. 8 (2011), no. 1, $19-28$.

[2] J. Adámek, H. Herrlich, and G. E. Strecker, Abstract and Concrete Categories: The Joy of Cats, Dover Publications (Mineola, New York), 2009.

[3] D. Aerts, E. Colebunders, A. van der Voorde, and B. van Steirteghem, State property systems and closure spaces: a study of categorical equivalence, Int. J. Theor. Phys. 38 (1999), no. 1, 359-385.

[4] D. Aerts, E. Colebunders, A. van der Voorde, and B. van Steirteghem, On the amnestic modification of the category of state property systems, Appl. Categ. Struct. 10 (2002), no. 5, 469-480.

[5] P. Baltazar, J. Ramos, and C. Sernadas, Probability and quantum institutions revisited, Tech. report, CLC, Department of Mathematics, Instituto Superior Técnico, Lisboa, Portugal, 2006, presented at WADT'06, La Roche en Ardenne, Belgium, June 1-3, 2006.

[6] B. Banaschewski and E. Nelson, Tensor products and bimorphisms, Canad. Math. Bull. 19 (1976), no. 4, 385-402.

[7] M. Barr, *-Autonomous Categories. With an appendix by Po-Hsiang Chu, Springer-Verlag, 1979.

[8] C. Caleiro, P. Mateus, A. Sernadas, and C. Sernadas, Quantum institutions, Algebra, Meaning, and Computation, Lecture Notes in Comput. Sci., vol. 4060, Springer, Berlin, 2006, pp. 50-64.

[9] J. T. Denniston, A. Melton, and S. E. Rodabaugh, Lattice-valued topological systems, Abstracts of the 30th Linz Seminar on Fuzzy Set Theory (U. Bodenhofer, B. De Baets, E. P. Klement, and S. Saminger-Platz, eds.), Johannes Kepler Universität, Linz, 2009, pp. 24-31.

[10] J. T. Denniston, A. Melton, and S. E. Rodabaugh, Interweaving algebra and topology: Lattice-valued topological systems, Fuzzy Sets Syst. 192 (2012), 58-103.

[11] J. T. Denniston, A. Melton, and S. E. Rodabaugh, Lattice-valued institutions, Abstracts of the 35th Linz Seminar on Fuzzy Set Theory (T. Flaminio, L. Godo, S. Gottwald, and E. P. Klement, eds.), Johannes Kepler Universität, Linz, 2014, pp. $44-46$.

[12] R. Diaconescu, Grothendieck Institutions, Appl. Categ. Structures 10 (2002), 338-402. 
[13] R. Diaconescu, Institution-Independent Model Theory, Birkhäuser Verlag, Basel, 2008.

[14] Y. Diers, Categories of algebraic sets, Appl. Categ. Struct. 4 (1996), no. 2-3, 329-341.

[15] Y. Diers, Affine algebraic sets relative to an algebraic theory, J. Geom. 65 (1999), no. 1-2, 54-76.

[16] Y. Diers, Topological geometrical categories, J. Pure Appl. Algebra 168 (2002), no. 2-3, $177-187$.

[17] H.-D. Ehrich and C. Caleiro, Specifying communication in distributed information systems, Acta Inf. 36 (2000), no. 8, 591-616.

[18] P. Eklund, M. Á. Galán, R. Helgesson, and J. Kortelainen, Fuzzy terms, Fuzzy Sets Syst. 256 (2014), $211-235$.

[19] P. Eklund, M. Á. Galán, J. Medina, M. Ojeda-Aciego, and A. Valverde, Set functors, L-fuzzy set categories, and generalized terms, Comput. Math. Appl. 43 (2002), no. 6-7, 693-705.

[20] P. Eklund, M. Á. Galán, J. Medina, M. Ojeda-Aciego, and A. Valverde, Similarities between powersets of terms, Fuzzy Sets Syst. 144 (2004), no. 1, 213-225.

[21] P. Eklund, M. Á. Galán, J. Medina, M. Ojeda-Aciego, and A. Valverde, Powersets of terms and composite monads, Fuzzy Sets Syst. 158 (2007), no. 23, 2552-2574.

[22] P. Eklund, J. Kortelainen, and L. N. Stout, Adding fuzziness to terms and powerobjects using a monadic approach, Fuzzy Sets Syst. 192 (2012), 104-122.

[23] J. Goguen and G. Roşu, Institution morphisms, Formal Asp. Comput. 13 (2002), no. 3-5, 274-307.

[24] J. A. Goguen and R. M. Burstall, Introducing institutions, Logics of Programs (Pittsburgh, Pa., 1983), Lecture Notes in Comput. Sci., vol. 164, Springer, Berlin, 1984, pp. 221-256.

[25] J. A. Goguen and R. M. Burstall, A study in the foundations of programming methodology: specifications, institutions, charters and parchments, Category Theory and Computer Programming (Guildford, 1985), Lecture Notes in Comput. Sci., vol. 240, Springer, Berlin, 1986, pp. 313-333.

[26] J. A. Goguen and R. M. Burstall, Institutions: abstract model theory for specification and programming, J. Assoc. Comput. Mach. 39 (1992), no. 1, 95-146.

[27] C. Guido, Fuzzy points and attachment, Fuzzy Sets Syst. 161 (2010), no. 16, 2150-2165.

[28] P. Hájek, Metamathematics of Fuzzy Logic, Dordrecht: Kluwer Academic Publishers, 1998.

[29] H. Herrlich and G. E. Strecker, Category Theory, 3rd ed., Sigma Series in Pure Mathematics, vol. 1, Heldermann Verlag, 2007.

[30] H. Hogbe-Nlend, Bornologies and Functional Analysis, Mathematics Studies, vol. 26, North-Holland Publishing Company, 1977.

[31] U. Höhle and A. P. Šostak, Axiomatic Foundations of Fixed-Basis Fuzzy Topology, Mathematics of Fuzzy Sets: Logic, Topology and Measure Theory (U. Höhle and S. E. Rodabaugh, eds.), The Handbooks of Fuzzy Sets Series, vol. 3, Dordrecht: Kluwer Academic Publishers, 1999, pp. 123-272.

[32] P. T. Johnstone, Stone Spaces, Cambridge University Press, 1982.

[33] F. W. Lawvere, Functorial Semantics of Algebraic Theories, Ph.D. thesis, Columbia University, 1963.

[34] B. Mayoh, Galleries and institutions, Tech. Report DAIMI PB-191, Aarhus University, 1985.

[35] T. Mossakowski, Representation, Hierarchies, and Graphs of Institutions, Ph.D. thesis, University of Bremen, 1996.

[36] J. Paseka, S. Solovyov, and M. Stehlík, Lattice-valued bornological systems, submitted to Fuzzy Sets Syst. (Special Issue: LINZ 2013).

[37] J. Paseka, S. Solovyov, and M. Stehlík, On the category of lattice-valued bornological vectors spaces, J. Math. Anal. Appl. 419 (2014), no. 1, 138-155.

[38] S. E. Rodabaugh, Categorical Foundations of Variable-Basis Fuzzy Topology, Mathematics of Fuzzy Sets: Logic, Topology and Measure Theory (U. Höhle and S. E. Rodabaugh, eds.), The Handbooks of Fuzzy Sets Series, vol. 3, Dordrecht: Kluwer Academic Publishers, 1999, pp. 273-388.

[39] S. E. Rodabaugh, Powerset Operator Foundations for Poslat Fuzzy Set Theories and Topologies, Mathematics of Fuzzy Sets: Logic, Topology and Measure Theory (U. Höhle and S. E. Rodabaugh, eds.), The Handbooks of Fuzzy Sets Series, vol. 3, Dordrecht: Kluwer Academic Publishers, 1999, pp. 91-116.

[40] A. Salibra and G. Scollo, A soft stairway to institutions, Recent Trends in Data Type Specification (Dourdan, 1991), Lecture Notes in Comput. Sci., vol. 655, Springer, Berlin, 1993, pp. 310-329.

[41] A. Sernadas, C. Sernadas, and J. M. Valença, A topological view on institutions, Tech. report, CLC, Department of Mathematics, Instituto Superior Técnico, Lisboa, Portugal, 1994.

[42] A. Sernadas, C. Sernadas, and J. M. Valença, A theory-based topological notion of institution, Recent Trends in Data Type Specification (E. Astesiano, G. Reggio, and A. Tarlecki, eds.), Lecture Notes in Computer Science, vol. 906, Springer Berlin Heidelberg, 1995, pp. 420-436.

[43] S. Solovjovs, Embedding topology into algebra, Abstracts of the 30th Linz Seminar on Fuzzy Set Theory (U. Bodenhofer, B. De Baets, E. P. Klement, and S. Saminger-Platz, eds.), Johannes Kepler Universität, Linz, 2009, pp. 106-110.

[44] S. Solovjovs, Categorically-algebraic topology, Abstracts of the International Conference on Algebras and Lattices (Jardafest), Charles University, Prague, 2010, pp. 20-22.

[45] S. Solovyov, Functorial semantics of topological theories, submitted to Iran. J. Fuzzy Syst.

[46] S. Solovyov, Localification procedure for affine systems, to appear in Cah. Topologie Géom. Différ. Catégoriques.

[47] S. Solovyov, Categorical foundations of variety-based topology and topological systems, Fuzzy Sets Syst. 192 (2012), $176-200$

[48] S. Solovyov, Categorically-algebraic topology versus universal topology, Fuzzy Sets Syst. 227 (2013), 25-45.

[49] S. Vickers, Topology via Logic, Cambridge University Press, 1989.

[50] S. Vickers, Geometric Logic in Computer Science, Theory and Formal Methods 1993 (G. Burn, S. Gay, and M. Ryan, eds.), Workshops in Computing, Springer London, 1993, pp. 37-54. 